\title{
Antibody responses to pertussis toxin display different kinetics after clinical Bordetella pertussis infection than after vaccination with an acellular pertussis vaccine
}

\author{
Tine Dalby, ${ }^{1}$ Jesper Westphal Petersen, ${ }^{2}$ Zitta B. Harboe ${ }^{1}$ \\ and Karen Angeliki Krogfelt ${ }^{1}$ \\ ${ }^{1}$ Department of Microbiological Surveillance and Research, Statens Serum Institut, \\ Copenhagen, Denmark \\ ${ }^{2}$ Bacterial Vaccine Development Department, Statens Serum Institut, Copenhagen, Denmark
}

Correspondence

Tine Dalby

tid@ssi.dk

Received 23 March 2010

Accepted 26 May 2010

\section{INTRODUCTION}

Pertussis has gained great attention in recent years, with immunization programmes in several countries being expanded to include not only older children and relevant health-care personnel, but also adolescents and adults in general (CDC, 2010; Haut Conseil de la Santé Publique, 2010; Bundesministerium für Gesundheit, 2009; Ständige Impfkommission, 2009; Nasjonalt Folkehelseinstitutt, 2007). Vaccines against Bordetella pertussis are based either on whole cells or on a number of acellular components, but common for all is the presence of pertussis toxin (PT). PT is unique to $B$. pertussis, and serological diagnosis based on

Abbreviations: TdaP-IPV, tetanus, diphtheria, acellular pertussis, inactivated polio combination vaccine; IQR, interquartile range; $\mathrm{PT}$, pertussis toxin.

Supplementary figures are available with the online version of this paper. detection of antibodies to PT is therefore very specific in addition to being highly sensitive (André et al., 2008; Baughman et al., 2004; de Melker et al., 2000). Serological diagnosis of pertussis has been introduced in many countries, and knowledge of the kinetics of the antibody responses to both vaccination and infection is crucial in order to interpret serological results correctly.

Several studies have described the decay of IgG antibodies to PT (IgG anti-PT antibodies) after vaccination (McIntyre et al., 2009; Hendrikx et al., 2009; Bailleux et al., 2008; Riffelmann et al., 2008; Edelman et al., 2007; Barreto et al., 2007; Hallander et al., 2005; Le et al., 2004; Keitel et al., 1999; Giuliano et al., 1998; Di Tommaso et al., 1997; Edwards et al., 1993), after infection (Heininger et al., 2004; Teunis et al., 2002; de Melker et al., 2000; Hodder et al., 2000; Giammanco et al., 1997) or both (Hallander et al., 2009; Isacson, 1997). Common for both types of 
response is the initial peak in antibody levels followed by a biphasic decay consisting of a rapid decay from the peak followed by a slower decay lasting for several years. However, the magnitudes of the responses reported from these studies vary widely both between and within the studies. From studies evaluating post-vaccination kinetics, the magnitude of the response was shown to differ between different vaccine types (Hendrikx et al., 2009; Keitel et al., 1999; Giuliano et al., 1998). From studies of the post-infection response, the magnitude of the response was shown to correlate with the type of pertussis vaccine received previously (Hallander et al., 2009; Giammanco et al., 1997).

Two studies from Sweden have previously compared postvaccination and post-infection IgG anti-PT antibody kinetics in children (Hallander et al., 2009; Isacson, 1997). To date, no studies on the comparison of post-vaccination and post-infection antibody kinetics in adults have been described. The aim of the present study was to describe the long-term IgG anti-PT antibody kinetics in Danish children and adults with bacteriologically confirmed $B$. pertussis infection and compare it to the long-term antibody kinetics from pertussis booster-vaccinated Danish adults.

\section{METHODS}

Study participants and sera. The kinetics of the anti-PT response was studied in individuals with bacteriologically confirmed infection with $B$. pertussis (by culture and/or PCR) as well as in individuals booster-vaccinated with a TdaP-IPV combination vaccine containing acellular PT (DiTeKiPol Booster; Statens Serum Institut). All involved participants were provided with a letter of information and signed a written consent before enrolment. Collection of samples from individuals with bacteriologically confirmed pertussis infection was performed by general practitioners and approved by The Danish College of General Practitioners (MPU 4-2007) and the Danish Data Protection Agency (2007-54-0061). The study was approved by the Scientific Ethical Committee for Copenhagen and Frederiksberg, Denmark (KF 01 318860). Collection of samples from individuals with confirmed pertussis was carried out from October 2006 to November 2009. Collection of samples from vaccinated individuals was carried out from October 2006 to October 2008. The Danish vaccination schedule at the time of the study consisted of three childhood vaccinations ( 3,5 and 12 months of age) and a pre-school booster at 5 years of age. There were no scheduled adolescent, adult or cocooning vaccinations.

Throughout the collection period, 237 individuals with confirmed pertussis were eligible for the study; 151 of those were contacted and 71 agreed to participate. Hence the study included serum samples from 71 individuals with bacteriologically confirmed infection with $B$. pertussis [30 males and 41 females, age 8-65 years, median age 21 years, interquartile range (IQR) 12-41 years), henceforth denoted 'patients'. Five patients were 8-9 years old, 22 were $10-14$ years old, 8 were 15-19 years old and 36 were 20 years or older. Only patients older than 7 years were included in the study in order to avoid interference by remaining antibodies from the pre-school booster. The diagnosis was made by IS481 insertion element PCR (van der Zee et al., 1993) and/or by culturing of the bacterium. Patients were asked to contact their practitioner for blood sampling as soon as possible after receiving the letter of invitation as well as 2, 5, 8, 11 and 14 months after the initial pertussis diagnosis. Forty patients delivered six samples, eight delivered five samples, eight delivered four samples and 15 delivered three or fewer. Patients were asked to rate the severity of the cough at the time of the first sample on a scale from 0 to $10 ; 60$ of the 71 patients reported the severity of cough with a median of 5.5 (IQR 4-7.1). Two individuals reported a history of pertussis 55 and 58 years previously, respectively. Other clinical characteristics were not reported. One individual reported the use of immunosuppressive drugs, but for the remaining 70 patients similar information was unavailable.

The study also included serum samples from 20 healthy adults employed at a vaccine-producing facility who due to safety precautions had to be booster-vaccinated against pertussis (8 males and 12 females, age 30-57, median age 39 years, IQR 33-47 years), henceforth denoted 'vaccinees'. The vaccine was a TdaP-IPV combination vaccine (DiTeKiPol Booster; Statens Serum Institut) containing $20 \mu \mathrm{g}$ acellular PT as toxoid as the sole pertussis antigen. The vaccinees were asked to deliver up to 13 blood samples at increasing intervals from 1 week after vaccination up to 2 years after vaccination. Three vaccinees delivered 13 samples, 7 delivered 12 samples, 5 delivered 11 samples, 2 delivered 10 samples and 3 delivered 7 samples or fewer. Pre-vaccination samples were available for 18 of the participants, and these samples had been taken a median of 5 months before vaccination (range 5-156 days, IQR 142156 days). One individual reported confirmed pertussis approximately 1.5 years prior to the initiation of the study. Otherwise the history of previous pertussis was unknown for the participants.

For both studies, the type and number of pertussis vaccines received in childhood was unknown; however, the Danish whole-cell pertussis vaccine was introduced in 1961 and substituted with the acellular pertussis vaccine in 1997. Vaccination coverage in Denmark has been high throughout the years (Andersen et al., 2007; Christensen \& Rønne, 1997; Nielsen \& Larsen, 1990; Nielsen \& Rønne, 1988). Hence, when testing for differences in the groups, the years 1961 and 1997 were used as limits when categorizing the participants. For the vaccinees, only three individuals were born before 1961 and valid statistical calculations were therefore impossible.

Detection of IgG anti-PT antibodies. IgG antibodies to PT were measured by indirect ELISA according to the method described previously (Dalby et al., 2009). Results were expressed as IU ml $\mathrm{m}^{-1}$ according to the WHO International Standard Pertussis Antiserum National Institute for Biological Standards and Control code 06/140. The Danish cut-off value for diagnostic purposes is set at $75 \mathrm{IU} \mathrm{ml}^{-1}$, as calculated from IgG anti-PT analysis of sera from 271 healthy Danish blood donors aged 18 years and older (mean plus 2 standard deviations from $\log _{10}$-transformed data plus an extra $20 \%$ margin as grey zone).

Calculation of antibody half-life. Antibody decay rates were calculated by use of linear regression on $\log _{2}$ transformed serological results in relation to days since the diagnosis (patients) or days since vaccination (vaccinees). Antibody half-life was expressed as the inverse reciprocal of the regression line slope (expressed in days).

Statistical analysis. Statistical analyses were performed using SAS statistical software (version 9.1.3). Due to the small sample size, nonparametric analyses by either Wilcoxon rank sum tests (two groups of data), Kruskal-Wallis tests (more than two groups of data) or Spearman rank-order correlations (continuous variables) were used.

\section{RESULTS AND DISCUSSION}

\section{Antibody-response profiles for patients}

The individual antibody-response profiles for the 71 patients are shown in Fig. 1 (also see Supplementary 


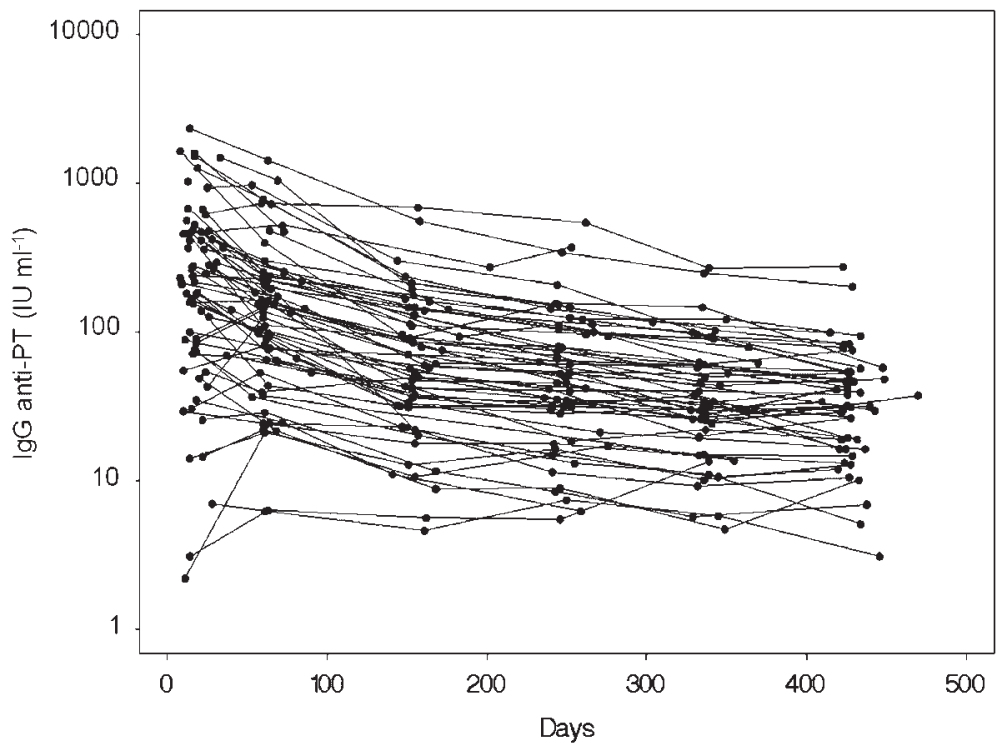

Fig. 1. Individual antibody decay profiles of $\lg \mathrm{G}$ anti-PT antibody concentrations (IU ml ${ }^{-1}$ ) for individuals with bacteriologically confirmed $B$. pertussis infection $(n=71)$. 'Days' are days since diagnosis and $\lg \mathrm{G}$ anti-PT results are shown on a $\log _{10}$ scale.

Figs S1 and S2 in JMM Online), and reverse cumulative response curves are shown in Fig. 2. Please note that the first sample should not be interpreted as an acute phase sample, as this sample was drawn some weeks after the

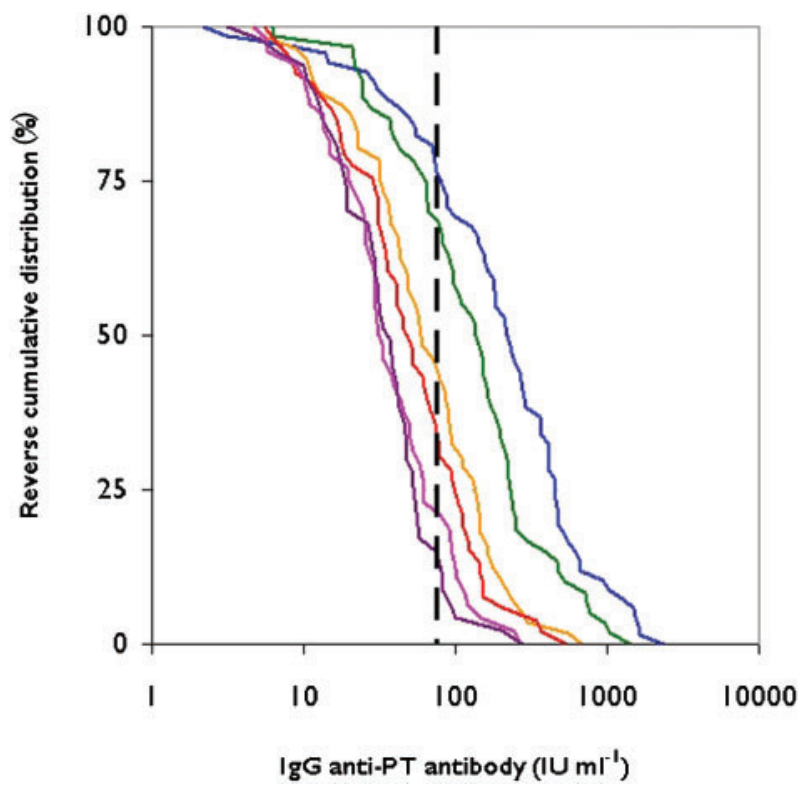

Fig. 2. Reverse cumulative distribution of $\lg G$ anti-PT antibody concentrations (IU ml${ }^{-1}$ ) for individuals with bacteriologically confirmed $B$. pertussis infection $(n=71)$. Blue, first sample some weeks after diagnosis ( $n=69)$; green, 2 months post-diagnosis ( $n=61)$; orange, 5 months post-diagnosis $(n=57)$; red, 8 months post-diagnosis $(n=54)$; pink, 11 months post-diagnosis $(n=49)$; purple, 14 months post-diagnosis $(n=48)$. The vertical line at $75 \mathrm{IU} \mathrm{ml} \mathrm{m}^{-1}$ marks the Danish diagnostic cut-off at serological diagnosis of pertussis. initial laboratory-confirmed diagnosis of pertussis was made.

The IgG anti-PT antibody response levels in individuals with pertussis infection varied in a broad range, but as shown in Fig. 1, the decay curves were roughly parallel. No differences in responses between different age groups were seen. Moreover, results from the youngest participants at 8 and 9 years of age were not distinguishable from the responses from the other age groups, thus indicating no particular effect of remaining antibodies from the preschool booster at that age. Such large variations in the magnitude of the antibody response have been described previously (Heininger et al., 2004; Teunis et al., 2002; de Melker et al., 2000; Hodder et al., 2000; Isacson, 1997), and similar parallel antibody decays have been observed (Teunis et al., 2002; de Melker et al., 2000). IgG anti-PT levels from the individual with reported use of immunosuppressive drugs were very low (the maximum value measured was $24 \mathrm{IU} \mathrm{ml}^{-1}$ ); however, antibody levels from seven other patients were similarly low (maximum values ranging from 7 to $35 \mathrm{IU} \mathrm{ml}^{-1}$ ).

The distribution of results in relation to time since diagnosis can be seen in Fig. 2. At the first sampling (median 17 days after diagnosis, range 8-63 days, IQR 1423 days), 53 of the available 69 samples (77\%) had antibody levels above the Danish diagnostic cut-off at $75 \mathrm{IU} \mathrm{ml}^{-1}$. Two individuals with a missing first sample were moreover positive at their first sampling, 2 months post-diagnosis. For two of the patients with a first-sample result below the cut-off ( 43 and $55 \mathrm{IU} \mathrm{ml}^{-1}$, respectively), the results at 2 months post-diagnosis were above the cutoff (125 and $81 \mathrm{IU} \mathrm{ml}^{-1}$, respectively), mimicking positive results at paired serology. In total, 57 of the 71 patients had antibody levels above the diagnostic cut-off at some point during the study. When omitting the results from the individual with reported use of immunosuppressive drugs, 
the sensitivity at single-point serology was $79 \%(55 / 70)$ and at paired serology was $81 \%(57 / 70)$. At the last sample 14 months after diagnosis (median 427 days after diagnosis, range 410-470 days, IQR 425-433 days), 8 of the available 48 samples (16.7\%) had antibody levels above the diagnostic cut-off.

The maximum achieved IgG anti-PT antibody level for each patient varied from 7 to $2300 \mathrm{IU} \mathrm{ml}^{-1}$ with a median of $217 \mathrm{IU} \mathrm{ml}^{-1}$ (IQR 85-467 IU ml ${ }^{-1}$ ). When correlating this maximum level to sex, age, severity of cough and assumed type of received childhood vaccine, none of these comparisons gave statistically significant differences. Only two of the 71 individuals had reported a previous episode of pertussis, and their antibody responses were not in any way distinguishable from those of the other 69 individuals. Also, it is very unlikely that only two of the 71 individuals had encountered pertussis previously.

\section{Antibody-response profiles for vaccinees}

The individual antibody-response profiles for the 20 vaccinees are shown in Fig. 3, and reverse cumulative response curves for a subset of the 13 samplings points are shown in Fig. 4.

As for the patients, the IgG anti-PT antibody-response levels varied over a broad range for the vaccinees, but as can be seen in Fig. 3, the decay curves were roughly parallel. Although some of the vaccinees had very low response levels, a distinct response curve with an initial increase, a peak and a slow decline was observed for all vaccinees. Previous studies have shown similar individual variations in the magnitude of the response (McIntyre et al., 2009; Hendrikx et al., 2009; Riffelmann et al., 2008; Edelman et al., 2007; Giuliano et al., 1998; Isacson, 1997), and parallel curves have also been observed (Di Tommaso et al., 1997).
The distribution of results in relation to time since vaccination can be seen in Fig. 4. Before vaccination, 3 of the 18 available samples (17\%) had IgG anti-PT antibody levels above the Danish diagnostic cut-off at $75 \mathrm{IU} \mathrm{ml}^{-1}$. The individual with the highest pre-vaccine level (104 IU $\mathrm{ml}^{-1}$ ) was also the individual who had reported an episode of pertussis less than 2 years prior to the study. Previous pertussis was not reported by the remaining two individuals; however, this does not exclude atypical or subclinical pertussis in the previous years. At 1 month post-vaccination (median 32 days, range 27-32 days), 12 of the 16 available samples $(75 \%)$ had antibody levels above the cut-off. Two years after vaccination (median 746 days, range 734-749 days), 6 of the 15 available samples $(40 \%)$ had antibody levels above the cut-off.

The maximum achieved antibody level for each vaccinee varied from 12 to $2500 \mathrm{IU} \mathrm{ml}^{-1}$ with a median of $183 \mathrm{IU}$ $\mathrm{ml}^{-1}$ (IQR 59-352 IU ml $\mathrm{ml}^{-1}$ ). When correlating this maximum level to sex, age and assumed type of received childhood vaccine, none of these comparisons gave statistically significant differences.

\section{Half-life for antibody responses}

Half-life was calculated only for data in the late-decay phase, i.e. after the first rapid decay from the peak. The shift in the biphasic curves from fast to slow decay was difficult to determine for each individual, and the range for the late phase in each group was accordingly chosen where the $\log _{2}-$ linear regression lines gave the best fit. For patients, half-life calculations were performed for the 5, 8, 11 and 14 month samples. For vaccinees, half-life calculations were performed for samples $\geqslant 90$ days after vaccination.

Fifteen patients had delivered either no sample or only one sample in the 5-14 month post-diagnosis period, and

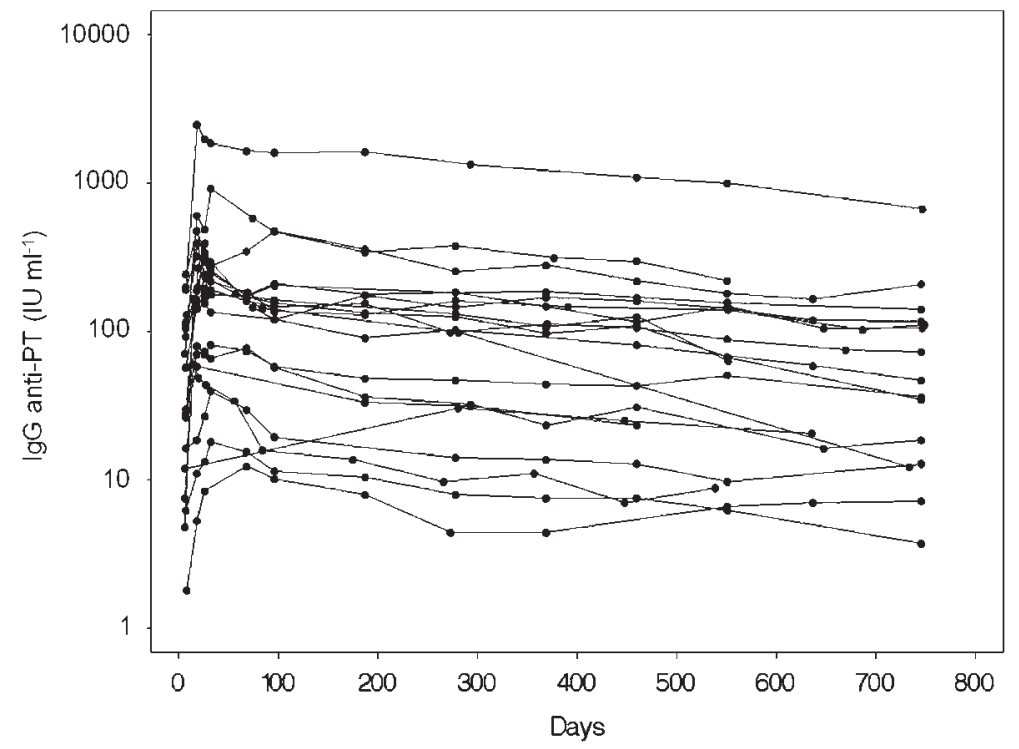

Fig. 3. Individual antibody decay profiles of IgG anti-PT antibody concentrations (IU ml ${ }^{-1}$ ) for individuals vaccinated with an acellular pertussis vaccine $(n=20)$. 'Days' are days since vaccination and $\lg \mathrm{G}$ anti-PT results are shown on a $\log _{10}$ scale. The results shown at day 0 represent pre-vaccine samples taken before vaccination. 


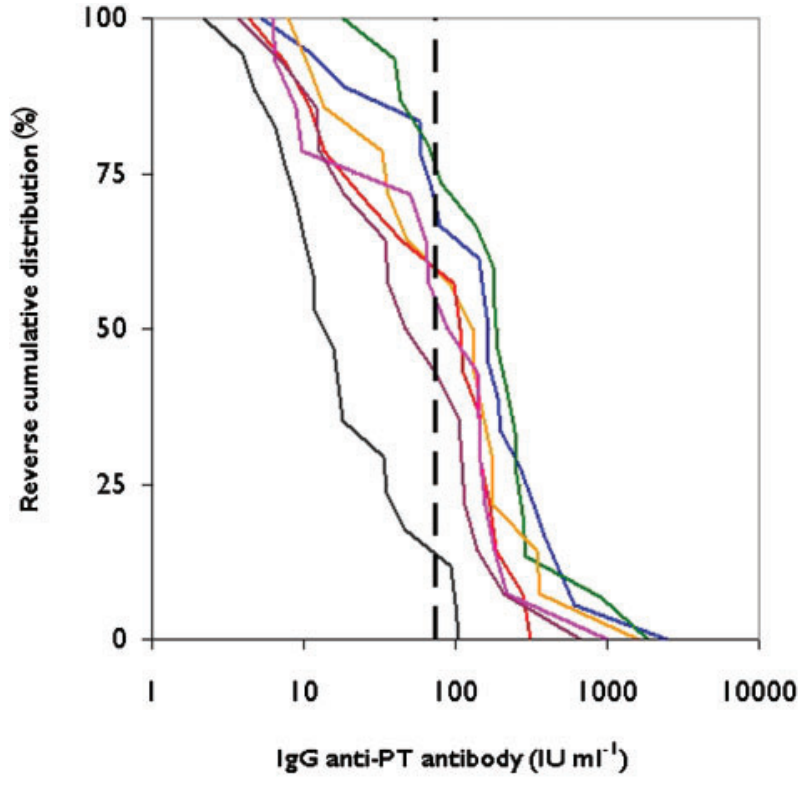

Fig. 4. Reverse cumulative distribution of $\lg G$ anti-PT antibody concentrations (IU ml${ }^{-1}$ ) for individuals vaccinated with an acellular pertussis vaccine $(n=20)$. Black, pre-vaccination sample $(n=18)$; blue, 2 weeks post-vaccination $(n=20)$; green, 1 month postvaccination ( $n=18)$; orange, 6 months post-vaccination $(n=18)$; red, 12 months post-vaccination $(n=20)$; pink, 18 months postvaccination ( $n=15)$; purple, 24 months post-vaccination $(n=15)$. The vertical line at $75 \mathrm{IU} \mathrm{ml}^{-1}$ marks the Danish diagnostic cut-off at serological diagnosis of pertussis.

consequently no half-life calculations were done. Data from individuals with only two samples after the initial peak were omitted from the half-life calculations (six patients, one vaccinee); therefore, only valid regression slopes from three or more datapoints were included. Data with almost horizontal decay were omitted from the halflife calculations (three vaccinees with antibody levels above the diagnostic cut-off). Data with very low antibody levels were omitted from the half-life calculations (five patients, two vaccinees). Data with nonlinear decay profiles were omitted from the half-life calculations (nine patients). For data with a single deviant result, this data point was omitted, leaving either three data points for the half-life calculation (seven patients) or five data points (one vaccinee). Half-life was consequently calculated for 36 patients and 14 vaccinees.

For the 36 patients, the calculated half-life ranged from 106 to 2032 days, with a median of 221 days (IQR 159314 days). The $R^{2}$ values for these linear regressions ranged from 0.70 to 0.9998 with a median of 0.94 .

For the 14 vaccinees, the calculated half-life ranged from 317 to 1285 days, with a median of 508 days (IQR 428616 days). The $R^{2}$ values for these linear regressions ranged from 0.71 to 0.9997 with a median of 0.89 . For the three individuals with high initial values, one had almost horizontal decay throughout the study and was omitted from the half-life calculations, but the calculated half-life for the two other individuals was 524 and 613 days, respectively. Hence, the high initial value did not influence the decay kinetics.

When correlating the half-life within each group with sex, age, assumed type of received childhood vaccine, severity of cough (patients) and highest achieved antibody level, none of these comparisons gave statistically significant differences. A previous study has shown a correlation of high IgG anti-PT levels with the length of spasmodic cough (Hallander et al., 2009), but in the current study we had no data on the length of the cough for the patients and similar comparisons were therefore not possible.

Using the obtained $\log _{2}$-linear equations for the IgG antiPT antibody decay for these $36+14$ individuals (patients and vaccinees, respectively), the time required for the IgG anti-PT antibody level to reach the Danish diagnostic cutoff at $75 \mathrm{IU} \mathrm{ml}{ }^{-1}$ could be calculated. For patients, the antibody levels were estimated to reach the cut-off within 2 years from point of diagnosis for all 36 individuals. The individual with the highest registered antibody level was estimated to reach the cut-off at 1.9 years post-diagnosis. For the remaining 35 patients without a valid $\log _{2}$-linear equation, a similar mathematical estimation is impossible; however, by visual estimation from the graphs it seems that none of the remaining patients will sustain an antibody level above $75 \mathrm{IU} \mathrm{ml} \mathrm{ml}^{-1} 2$ years after diagnosis. For vaccinees, 5 of the 14 individuals with valid half-life calculations had antibody levels so high that more than 2 years from point of vaccination are estimated for the antibody level to reach the cut-off: the longest expected time is estimated to be 6.6 years (the other four values are 5.3 years, 3.8 years, 3.5 years and 2.8 years). For the remaining 15 vaccinees, 13 reached the cut-off sooner than 2 years, and the last 2 had almost horizontal decay profiles above the cut-off making estimates impossible.

A number of other studies on both post-infection and post-vaccination antibodies have also been concerned with describing the kinetics of the IgG anti-PT antibody responses. Some have included mathematical approximations (Bailleux et al., 2008; Hallander et al., 2005; Le et al., 2004; Teunis et al., 2002; de Melker et al., 2000), or described the decay in terms of percentages (Riffelmann et al., 2008; Edelman et al., 2007; Heininger et al., 2004; Le et al., 2004), and others again have made graphical interpretations (Hallander et al., 2009; de Melker et al., 2000; Di Tommaso et al., 1997). Although Teunis et al. (2002) and de Melker et al. (2000) made mathematical models based on individual data, most approaches have used calculations or models based on either geometric mean or median results instead of on the individual response curves. The very large variations in the magnitude of the individual responses observed in our study, as well as in several other studies, emphasize the importance of using calculations based on each individual, both for patients and 
vaccinees. Estimating the percentage decay is similar to our approach, but only the study by Edelman et al. (2007) described the late-phase decay (from 3 to 5 years postvaccination). Three other studies by Riffelmann et al. (2008), Heininger et al. (2004) and Le et al. (2004) described the percentage decay relative to the peak level. Such percentages can be very difficult to compare since these calculations require a sample on the exact maximum of the peak to be valid for comparisons (and peak samples are seldom from the exact maximum). Also, the percentage reductions include both parts of the biphasic decay, and the initial rapid decay will therefore have a different impact on the results depending on the length of follow-up.

\section{Comparisons between patients and vaccinees}

We observed very large variations in the magnitudes of the IgG anti-PT response in both patients and vaccinees: an approximate 300 -fold difference between the lowest and the highest responder was seen among patients, and an approximate 200 -fold difference was seen among vaccinees. When comparing the maximum achieved antibody levels for individuals between patients and vaccinees, no statistically significant difference was found.

When comparing antibody decay half-life for patients and vaccinees, a statistically significant difference was found $(P<0.0001)$. A scatter-plot of these distributions is shown in Fig. 5. Although half-life could not be calculated for all the 91 participants in the study, the validity of the results is illustrated by Figs 1 and 3 showing uniform decay within the two groups.

Thus, the decay profiles of IgG anti-PT antibodies in Danish subjects after a $B$. pertussis infection or after acellular vaccination against pertussis are not identical. This is in contrast to results from studies with Swedish children (Hallander et al., 2009) and German adults (Heininger et al., 2004). However, both of those studies made the conclusions based on either median or geometric mean levels alone, and the individual decay profiles were not described. Even though sex and age showed no significant correlations in the present study to either the highest achieved IgG anti-PT antibody level or to the antibody decay half-life, other factors are likely to influence the antibody decay kinetics, making assumptions based on geometric means uncertain. Such factors could be: type(s) of childhood pertussis vaccine, number of previous pertussis infections, the genetic profile of the bacteria in such previous infections as well as general differences in the individual host response.

Although we found large individual variations in the magnitude of the antibody response in each of the two groups, the decay profiles were significantly different with the antibody decay after infection being approximately twice as fast as that after vaccination. While all of the patients with valid regression calculations were expected to have IgG anti-PT antibody levels below the Danish

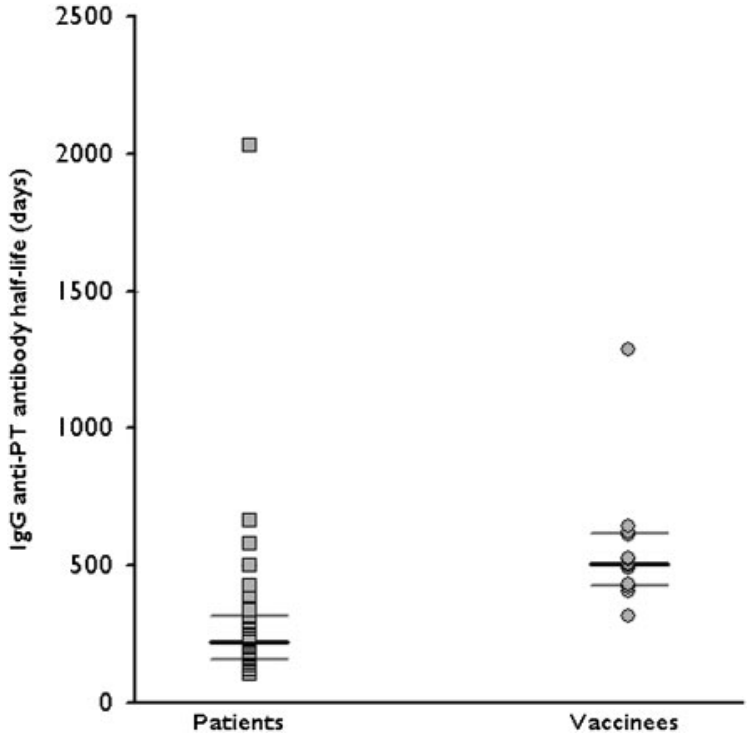

Fig. 5. IgG anti-PT antibody half-life calculated by linear regression on $\log _{2}$ transformed data for the longitudinal data shown in Fig. 1 and Fig. 3. Squares, data from 36 individuals with laboratory-confirmed pertussis 5-14 months post-diagnosis. Circles, data from 14 individuals vaccinated with an acellular pertussis vaccine 90 days -2 years post-vaccination. Median levels are shown as bold black horizontal lines; $25 \%$ and $75 \%$ quantiles are shown as grey horizontal lines.

diagnostic cut-off 2 years after diagnosis, at least $25 \%$ of the vaccinees would require more than 2 years for the antibody levels to reach this cut-off.

The results of this study emphasize the need for careful considerations when deciding which individuals are suitable for serological diagnosis of pertussis due to the potential presence of antibodies from a previous pertussis infection or vaccination. A limit of 2 years since a previous pertussis infection is probably just barely enough to allow the infection antibodies to decrease below the diagnostic cut-off. A limit of 2 years from vaccination, however, seems to be too short a time span, at least for adults vaccinated with the Danish acellular TdaP-IPV vaccine; here a limit of at least 6 years since vaccination would be more accurate. Although the half-life of IgG anti-PT antibody decay after $B$. pertussis infection and after acellular pertussis vaccination is statistically different, half-life can probably not be used to decide whether an observed decrease in antibody levels between two samples originates from infection or vaccination. In Denmark, at present there are no adolescent, adult or cocooning vaccination strategies for pertussis. Thus, single-serum serological diagnosis of Danish individuals older than 8 years will be possible without interference from vaccination-induced antibodies. However, in countries with enhanced vaccination strategies, the vaccination status of individuals suspected of having pertussis should be carefully evaluated before serology can be used for diagnosis. 
Our study included sera collected from Danish individuals and a Danish vaccine, and extrapolations of our results to individuals belonging to other age groups or to studies of other types of pertussis vaccines or from other countries should therefore be made with great caution. Also, although a significant difference in antibody half-life was seen between patients and vaccinees, the study included data from only 91 individuals and the results are therefore only indicative of the true nature of these antibody responses.

In conclusion, we found that the kinetics of IgG anti-PT antibody response after infection with $B$. pertussis and after acellular vaccination against pertussis are not identical. Although both types of antibody decay show an initial peak followed by a slower decay, the decay of post-infection antibodies is more than twice as fast as that for postvaccination antibodies.

\section{ACKNOWLEDGEMENTS}

We would like to thank all the participants in the study for donation of blood samples, and the contribution from the general practitioners is also greatly valued. We are also very grateful for assistance provided by Ms Pernille Bangsgaard Ildved, Mr Asger Kjærgaard Mortensen, Ms Vita Skov and Ms Jette Heron. Ms Charlotte Sværke Jørgensen is thanked for fruitful discussions during this study. Sera from individuals with diagnosed pertussis were collected via the general practitioners with support from 'Forskningsfonden for Almen Praksis' (case no. 585-04/2072) and the following Danish regions: Sjælland, Midt, Syddanmark and Nordjylland.

\section{REFERENCES}

Andersen, P. H., Valentiner-Branth, P., Glismann, S., Christiansen, A. H. \& Simonsen, J. B. (2007). DTap-IPV/Hib vaccination: coverage by the end of 2005. EPI-NEWS No. 8 (www.ssi.dk).

André, P., Caro, V., Njamkepo, E., Wendelboe, A. M., Van Rie, A. \& Guiso, N. (2008). Comparison of serological and real-time PCR assays to diagnose Bordetella pertussis infection in 2007. J Clin Microbiol 46, 1672-1677.

Bailleux, F., Coudeville, L., Kolenc-Saban, A., Bevilacqua, J., Barreto, L. \& André, P. (2008). Predicted long-term persistence of pertussis antibodies in adolescents after an adolescent and adult formulation combined tetanus, diphtheria, and 5-component acellular pertussis vaccine, based on mathematical modeling and 5-year observed data. Vaccine 26, 3903-3908.

Barreto, L., Guasparini, R., Meekison, W., Noya, F., Young, L. \& Mills, E. (2007). Humoral immunity 5 years after booster immunization with an adolescent and adult formulation combined tetanus, diphtheria, and 5-component acellular pertussis vaccine. Vaccine 25, 81728179.

Baughman, A. L., Bisgard, K. M., Edwards, K. M., Guris, D., Decker, M. D., Holland, K., Meade, B. D. \& Lynn, F. (2004). Establishment of diagnostic cutoff points for levels of serum antibodies to pertussis toxin, filamentous hemagglutinin, and fimbriae in adolescents and adults in the United States. Clin Diagn Lab Immunol 11, 1045-1053.

Bundesministerium für Gesundheit (2009). Evidenz-basierte Empfehlungen des Obersten Sanitätsrates. Impfplan 2009 Österreich. http://www.bmgfj.gv.at/.

CDC (2010). Recommended adult immunization schedule - United States, 2010. MMWR 59, 1-4.
Christensen, M. \& Rønne, T. (1997). Coverage of the childhood vaccination programme 1988-1996. EPI-NYT Uge 47 (www.ssi. dk) (in Danish).

Dalby, T., Seier-Petersen, M., Kristiansen, M. P., Harboe, Z. B. \& Krogfelt, K. A. (2009). Problem solved: a modified enzymelinked immunosorbent assay for detection of human antibodies to pertussis toxin eliminates false-positive results occurring at analysis of heat-treated sera. Diagn Microbiol Infect Dis 63, 354360.

de Melker, H. E., Versteegh, F. G. A., Conyn-van Spaendonck, M. A. E., Elvers, L. H., Berbers, G. A. M., van der Zee, A. \& Schellekens, J. F. P. (2000). Specificity and sensitivity of high levels of immunoglobulin $\mathrm{G}$ antibodies against pertussis toxin in a single serum sample for diagnosis of infection with Bordetella pertussis. J Clin Microbiol 38, 800-806.

Di Tommaso, A., Bartalini, M., Peppoloni, S., Podda, A., Rappuoli, R. \& De Magistris, M. T. (1997). Acellular pertussis vaccines containing genetically detoxified pertussis toxin induce long-lasting humoral and cellular responses in adults. Vaccine 15, 1218-1224.

Edelman, K., He, Q., Mäkinen, J., Sahlberg, A., Haanperä, M., Schuerman, L., Wolter, J. \& Mertsola, J. (2007). Immunity to pertussis 5 years after booster immunization during adolescence. Clin Infect Dis 44, 1271-1277.

Edwards, K. M., Decker, M. D., Graham, B. S., Mezzatesta, J., Scott, J. \& Hackell, J. (1993). Adult immunization with acellular pertussis vaccine. JAMA 269, 53-56.

Giammanco, A., Taormina, S., Genovese, M., Mangiaracina, G., Giammanco, G. \& Chiarini, A. (1997). Serological responses to infection with B. pertussis. Dev Biol Stand 89, 213-220.

Giuliano, M., Mastrantonio, P., Giammanco, A., Piscitelli, A., Salmaso, S. \& Wassilak, S. G. F. (1998). Antibody responses and persistence in the two years after immunization with two acellular vaccines and one whole-cell vaccine against pertussis. J Pediatr 132, 983-988.

Hallander, H. O., Gustafsson, L., Ljungman, M. \& Storsaeter, J. (2005). Pertussis antitoxin decay after vaccination with DTPa. Response to a first booster dose $31 / 2-61 / 2$ years after the third vaccine dose. Vaccine 23, 5359-5364.

Hallander, H. O., Ljungman, M., Storsaeter, J. \& Gustafsson, L. (2009). Kinetics and sensitivity of ELISA IgG pertussis antitoxin after infection and vaccination with Bordetella pertussis in young children. APMIS 117, 797-807.

Haut Conseil de la Santé Publique (2010). Calendrier vaccinal détaillé 2009. http://www.sante-jeunesse-sports.gouv.fr/.

Heininger, U., Cherry, J. D. \& Stehr, K. (2004). Serologic response and antibody-titer decay in adults with pertussis. Clin Infect Dis 38, 591594.

Hendrikx, L. H., Berbers, G. A., Veenhoven, R. H., Sanders, E. A. M. \& Buisman, A.-M. (2009). IgG responses after booster vaccination with different pertussis vaccines in Dutch children 4 years of age: effect of vaccine antigen content. Vaccine 27, 6530-6536.

Hodder, S. L., Cherry, J. D., Mortimer, E. A., Jr, Ford, A. B., Gornbein, J. \& Papp, K. (2000). Antibody responses to Bordetella pertussis antigens and clinical correlations in elderly community residents. Clin Infect Dis 31, 7-14.

Isacson, J. (1997). Studies of pertussis epidemiology and of serum antibody responses to Bordetella pertussis antigens after disease and vaccination. Doctoral Thesis, Department of Pediatrics, SU/Östra sjukhuset, Göteborg University, Sweden.

Keitel, W. A., Muenz, L. R., Decker, M. D., Englund, J. A., Mink, C. M., Blumberg, D. A. \& Edwards, K. M. (1999). A randomized clinical trial of acellular pertussis vaccines in healthy adults: dose-response 
comparisons of 5 vaccines and implications for booster immunization. J Infect Dis 180, 397-403.

Le, T., Cherry, J. D., Chang, S.-J., Knoll, M. D., Lee, M. L., Barenkamp, S., Bernstein, D., Edelman, R., Edwards, K. M. \& other authors (2004). Immune responses and antibody decay after immunization of adolescents and adults with an acellular pertussis vaccine: the APERT Study. J Infect Dis 190, 535-544.

Mclntyre, P. B., Burgess, M. A., Egan, A., Schuerman, L. \& Hoet, B. (2009). Booster vaccination of adults with reduced-antigen-content diphtheria, Tetanus and pertussis vaccine: immunogenicity 5 years post-vaccination. Vaccine 27, 1062-1066.

Nasjonalt Folkehelseinstitutt (2007). Vaksinasjonsboka. http://www. fhi.no/

Nielsen, A. \& Larsen, S. O. (1990). [Whooping cough epidemiology in Denmark prior to and after the introduction of whooping cough vaccination. Protective effect of the vaccine and herd immunity] (in Danish). Ugeskr Laeger 152, 597-604.
Nielsen, A. \& Rønne, T. (1988). Coverage of the childhood vaccination programme 1979-1986. EPI-NYT Uge 21 (www.ssi.dk) (in Danish).

Riffelmann, M., Littmann, M., Hülße, C. \& Wirsing von König, C. H. (2008). Antibody decay after immunisation of health-care workers with an acellular pertussis vaccine. Eur J Clin Microbiol Infect Dis 28, 275-279.

Ständige Impfkommission (2009). Mitteilung der Ständigen Impfkommission am Robert Koch-Institut. Empfehlungen der Ständigen Impfkommission (STIKO) am Robert Koch-Institut/ Stand: Juli 2009. Epidemiol Bull 30, 279-298.

Teunis, P. F., van der Heijden, O. G., de Melker, H. E., Schellekens, J. F., Versteegh, F. G. \& Kretzschmar, M. E. (2002). Kinetics of the IgG antibody response to pertussis toxin after infection with $B$. pertussis. Epidemiol Infect 129, 479-489.

van der Zee, A., Agterberg, C., Peeters, M., Schellekens, J. \& Mooi, F. R. (1993). Polymerase chain reaction assay for pertussis: simultaneous detection and discrimination of Bordetella pertussis and Bordetella parapertussis. J Clin Microbiol 31, 2134-2140. 